J. Gynäkol. Endokrinol. AT 2019 · 29:39-44 https://doi.org/10.1007/s41974-019-0091-y

Online publiziert: 11. Juni 2019

(c) Der/die Autor(en) 2019

\section{Helge Binder}

Universitätsklinik für Gynäkologie, Geburtshilfe und Gynäkologische Endokrinologie, Kepler Universitätsklinikum GmbH, Med Campus IV., Linz, Österreich

\title{
Kontrazeption über 40 - was ist zweckmäßig und wie lange ist sie sinnvoll
}

Der Eintritt der Menopause bzw. dieletzte Periode sind von wichtigem klinischem und öffentlichem Interesse, da dieses Alter eine spezifische Zäsur im Alter einer Frau darstellt. Viel von unserem Wissen und Verständnis der Menopause beruht zu großen Teilen auf Untersuchungen an kaukasischen Frauen. Zudem sind viele der Studien klinischer Natur und stammen weniger aus epidemiologischen Daten [1]. Frauen, die in dieses Alter kommen, bewegen sich im Spannungsfeld der zu Ende gehenden reproduktiven Phase, von möglichen Krankheiten oder Beschwerden sowie der meist abgeschlossenen Familienplanung. Sollte es dann in dieser Phase zu Schwangerschaften kommen, kann das unabhängig von der Belastung der Spätgebärenden in der Schwangerschaft auch zu vermehrten Aborten kommen. Immerhin beträgt die Geburtenrate in einem Industrieland wie Kanada bei 40 - bis 44 -
Jährigen 8,4 Geburten pro 1000 Frauen. Diese sinkt dann auf 0,2 pro 1000 bei Frauen, die 45 Jahre und älter waren. Dies im Vergleich zu 107,4 Geburten pro 1000 Frauen bei den 30- bis 34-Jährigen. Die medizinischen Risiken einer ungeplanten Schwangerschaft sind deutlich größer für ältere Frauen im Vergleich zu jüngeren Frauen. Deswegen sollten die Risiken einer Kontrazeption gegen die Risiken einer Schwangerschaft abgewogen werden. Zusätzlich sollten die effektivsten kontrazeptiven Methoden angewandt werden, um die medizinischen Risiken einer ungeplanten Schwangerschaft bei älteren Frauen zu reduzieren [2].

Bedingt durch die veränderten Hormone leiden Frauen bereits in der Prämenopause und in der Perimenopause mehr oder weniger an vasomotorischen Symptomen, Stimmungsschwankungen, irregulären oder verstärkten Monatsblutungen (• Abb. 1 und 2). Ärzte müssen all dies bedenken, wenn sie den Ratsuchenden aus dieser Bevölkerungsgruppe eine adäquate kontrazeptive Option anbieten möchten [3].

Wie viele Frauen sind von dieser Fragestellung und Problematik betroffen? In der Bundesrepublik Deutschland sind nach einer Erhebung von 2012 ca. 10.624.000 40- bis 59-Jährige betroffen [6]. In Österreich ist die Zahl deutlich geringer mit 1.010.000 Frauen. Auch der Arzt findet sich in einem Spannungsfeld zwischen den Wünschen der Frau und dem eigenen Anspruch einer möglichst umfassenden Beratung hinsichtlich der geeigneten Kontrazeption bei älteren Frauen. Grundsätzlich wünschen Frauen über 35 bzw. 40 Jahre, befeuert durch entsprechende Presseartikel, möglichst eine hormonfreie Kontrazeption. Wie verhält es sich aber tatsächlich mit den Risiken oraler Kontrazeptiva bzw. der hormonellen Behandlung in der Peri-

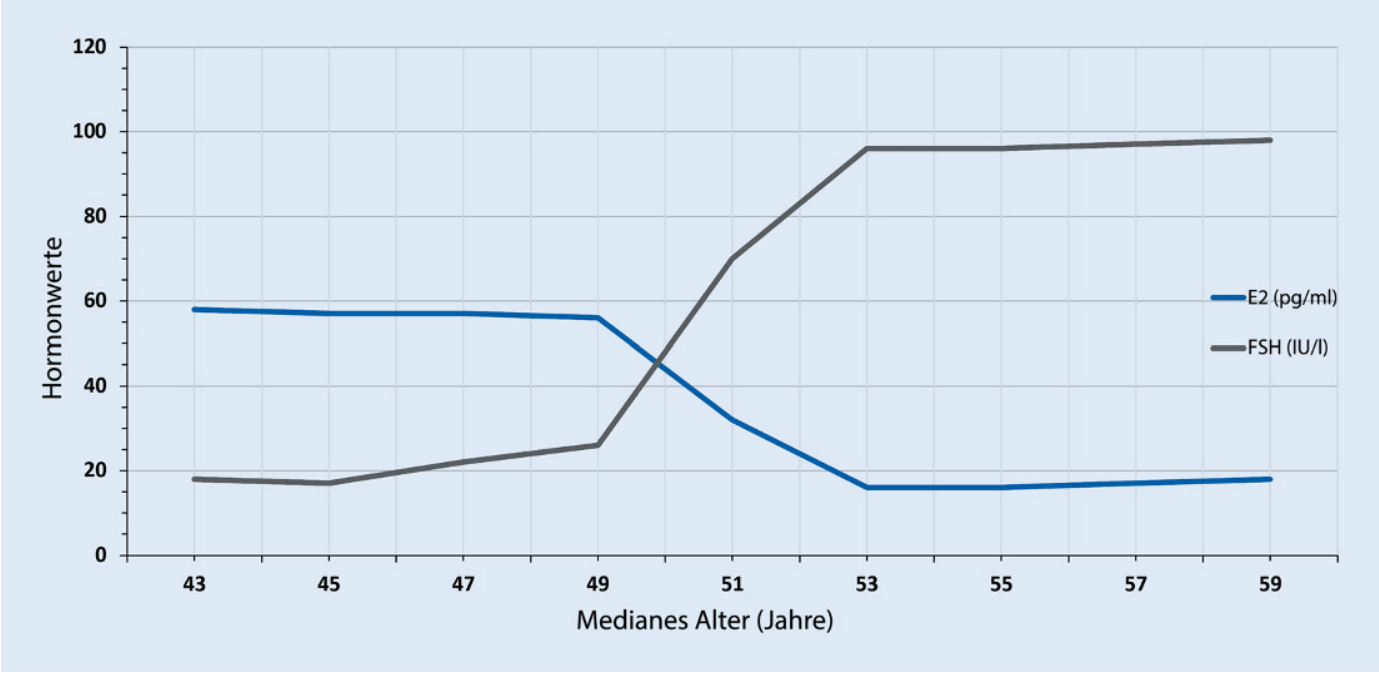

Abb. $1 \triangleleft$ Hormonverlauf in der Perimenopause. Östrogen und follikelstimulierendes Hormon (FSH). (Abgeändert nach [4]) 
und Postmenopause. Diese Problematik wurde Anfang der 2000er-Jahre in vielen wissenschaftlichen Publikationen, die Hormone verteufelt haben, aber auch in der Laienpresse als sehr dramatisch dargestellt (• Abb. 3; [7, 8]). Mittlerweile konnten durch Spätauswertungen der ursprünglichen WHI-Studie die Risiken relativiert werden. Es zeigt sich, dass die Hormontherapie einen positiven Einfluss auf die Inzidenz von bestimmten Karzinomen hat und einen erheblichen Vorteil auf die Inzidenz von Diabetes und Osteopenie oder Osteoporose zeigt. Die Zahl der Schlaganfälle, Lungenembolien und tiefen Beinvenenthrombosen wird durch Hormone negativ beeinflusst, während die koronaren Herzerkrankungen durch Östrogenmonotherapie positiv beeinflusst werden. Insgesamt sinkt die Gesamtmortalität.

Das orale Kontrazeptivum ist das häufigste Verhütungsmittel in Industrieländern. Neben der kontrazeptiven Wirkung haben orale Kontrazeptiva auch nichtkontrazeptive Gesundheitsvorteile, wie die Verbesserung von Akne, oder eine regulierende Wirkung auf Blutungsstörungen oder Dysmenorrhö [11-13]. Jedoch ist die Nutzung von oralen Kontrazeptiva nicht ohne Risiken. Einige Studien zeigen Nebenwirkungen wie tiefe Beinvenenthrombose, Myokardinfarkte und Schlaganfälle [14-16]. Bei den kombinierten oralen Kontrazeptiva werden in Bezug auf Krebserkrankungen immer wieder kontrovers Einflüsse diskutiert. Im Rahmen einer Metaanalyse wurde für bestimmte und häufige Krebserkrankungen der Frau eine positive Wirkung auf die Inzidenz des Endometriumkarzinoms und des kolorektalen Karzinoms gefunden. Im Rahmen dieser zitierten Metaanalyse [17], wurden 44 Studien zur Inzidenz von Brustkrebs unter OC-Nutzern, 12 hinsichtlich der Wirkung auf Zervixkarzinome, 11 auf kolorektale Karzinome und 9 Studien, die die Wirkung von OC auf die Inzidenz des Endometriumkarzinoms aufzeigten, ausgewertet (• Abb. 4). Neben den offensichtlichen Risiken bezüglich einer malignen Erkrankung treten im höheren Alter venöse und arterielle thromboembolische Erkrankungen häufiger auf, reine progesteronhaltige kontrazeptive

J. Gynäkol. Endokrinol. AT 2019 · 29:39-44 https://doi.org/10.1007/s41974-019-0091-y (c) Der/die Autor(en) 2019

\section{H. Binder}

\section{Kontrazeption über 40 - was ist zweckmäßig und wie lange ist sie sinnvoll}

\section{Zusammenfassung}

Frauen über 40 Jahre sind sexuell aktiv und benötigen eine effektive Kontrazeption, um Schwangerschaften zu vermeiden. Diese Altersgruppe hat andere Erfordernisse, die die Wahl der richtigen kontrazeptiven Methoden beeinflussen. Diese Altersgruppe hat möglicherweise bereits perimenopausale Symptome oder veränderte Blutungsmuster, die mit hormonellen Kontrazeptiva beeinflusst werden können. Zusätzlich treten bei Frauen über 40 häufiger Krankheiten auf. Dadurch sind bestimmte Kontrazeptiva unter Umständen nicht oder nicht mehr geeignet. Zudem gibt es generelle Unterschiede im Vergleich zu jüngeren Frauen, bedingt durch die niedrigere Schwangerschaftsrate, die ver- änderte Häufigkeit des Geschlechtsverkehrs oder höhere Compliance bei der Anwendung von Kontrazeptiva. Diese Übersicht beleuchtet die Auswahl der geeigneten Kontrazeptiva bei dieser Altersgruppe und wann die Kontrazeption im späteren Alter beendet werden sollte. Die meisten Empfehlungen basieren auf Leitlinien und auf systematischen Reviews. Diese Empfehlungen sind in einem Algorithmus zusammengefasst.

Schlüsselwörter

Blutungsstörungen · Orale Kontrazeptiva - Perimenopausale Symptome . Spätschwangerschaften $\cdot$ Hormone

\section{Contraception over 40: what is appropriate and for how long}

\section{Abstract}

Women over 40 years of age are sexually active and require effective contraception to avoid pregnancies. However, these women have special requirements that affect the choice of the appropriate contraceptive method. This age group may experience perimenopausal symptoms and altered bleeding patterns, which can be managed with hormonal contraceptives. In addition, these women may suffer from medical conditions that render certain contraceptive methods inappropriate. There are also differences compared to younger women due to lower fecundity, lower frequency of sexual intercourse and better compliance using contraceptives. This article outlines the benefits of appropriate contraceptive methods in women over 40 and when to stop contraception in later ages. Most of the recommendations are based on guidelines or systematic reviews and a comprehensive algorithm regarding the choice of methods is shown.

\section{Keywords}

Bleeding disorders $\cdot$ Perimenopausal symptoms · Oral contraceptives · Late-age pregnancies $\cdot$ Hormones 


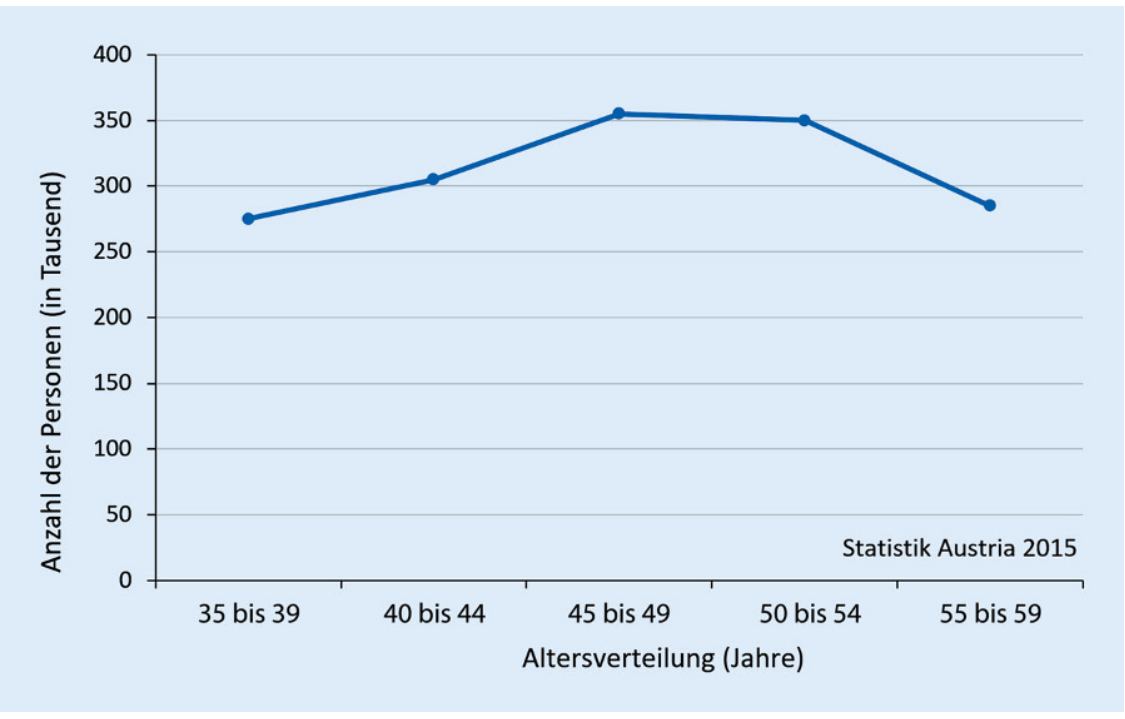

Abb. 2 ^ Zahl der betroffenen Frauen prämenopausal bzw. prämenopausal in Österreich [5]
Inzidenz mit höherem Alter und bei zusätzlichen Risikofaktoren wie Übergewicht, Rauchen und Bluthochdruck [21]. Neben den bekannten und weniger bekannten negativen Wirkungen von Kontrazeptiva in dieser Altersgruppe gibt es aber auch nichtkontrazeptive Vorteile. Je nach Wahl des geeigneten Kontrazeptivums können eine Hypermenorrhö oder vegetative bzw. vasomotorische Symptome oder die abnehmende Knochendichte erfolgreich kontrolliert bzw. behandelt werden (siehe dazu • Tab. 1).

Was empfehlen nun die Leitlinien. $\mathrm{Zu}$ diesem Thema gibt es keine deutschsprachigen Leitlinien, aber eine Vielzahl von Subgruppenempfehlungen. Die WHO gibt evidenzbasierte Ratschläge hinsicht-
Methoden scheinen das Risiko venöser thromboembolischer Ereignisse nicht zu erhöhen, weshalb sie für ältere, aber auch durch Fettleibigkeit oder andere medizinische Komorbiditäten beeinträchtigte Frauen geeigneter wären. In einer multinationalen fallkontrollierten Studie wurde kein erhöhtes Risiko von venösen Thromboembolien oder Myokardinfarkten gefunden. In einer anderen prospektiven Kohortenstudie über 15 Jahre konnte keine Erhöhung von arteriellen thromboembolischen Ereignissen durch ausschließlich progesteronhaltige kontrazeptive Methoden (IUD, Implantate oder Pillen) gefunden werden, verglichen zu Nichtnutzern [18, 19]. Der Gebrauch von östrogenhaltigen Kontrazeptiva erhöht das Risiko von venösen und arteriellen thromboembolischen Ereignissen. Weitere Risikofaktoren sind in diesem Zusammenhang das Alter, Übergewicht, Rauchen, Diabetes, Bluthochdruck, Migräne sowie genetisch bedingte Thrombophilien. Deswegen gilt seit Längerem schon grundsätzlich, dass östrogenhaltige Methoden bei Frauen über 40, die an kardiovaskulären Erkrankungen leiden, mit Vorsicht eingesetzt werden sollten [20]. Die Inzidenz, eine venöse Thromboembolie $\mathrm{zu}$ erleiden, beträgt bei Frauen im reproduktiven Alter 5-10 auf 10.000 Frauenjahre. Bei Nutzerinnen der kombinierten, also östrogenhaltigen Kontrazeptiva verdoppelt sich dieses Risiko. Zudem steigt die
Hier steht eine Anzeige.

\section{Springer}




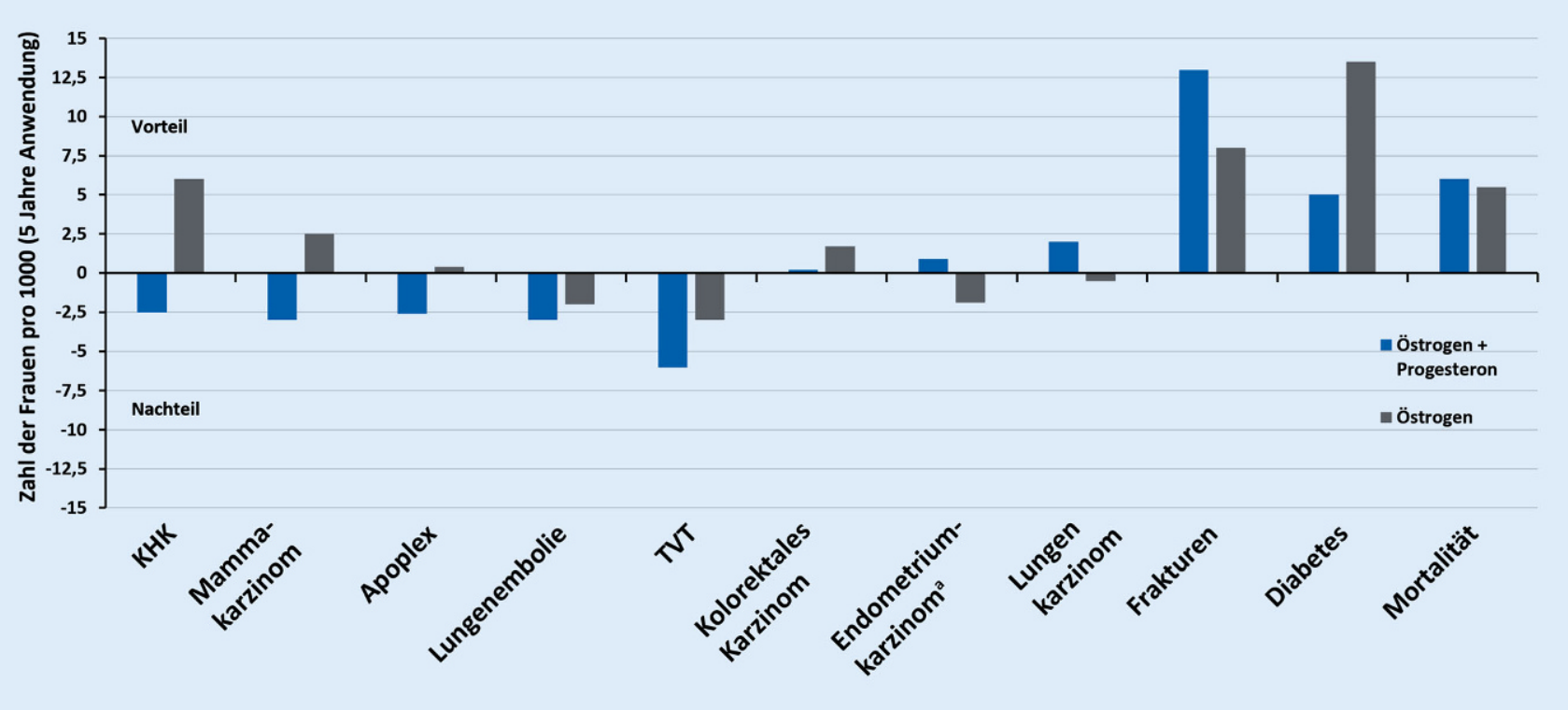

Abb. $3 \Delta$ Risiken und Vorteile durch die Hormontherapie [9]. 2Umgerechnet nach [10]

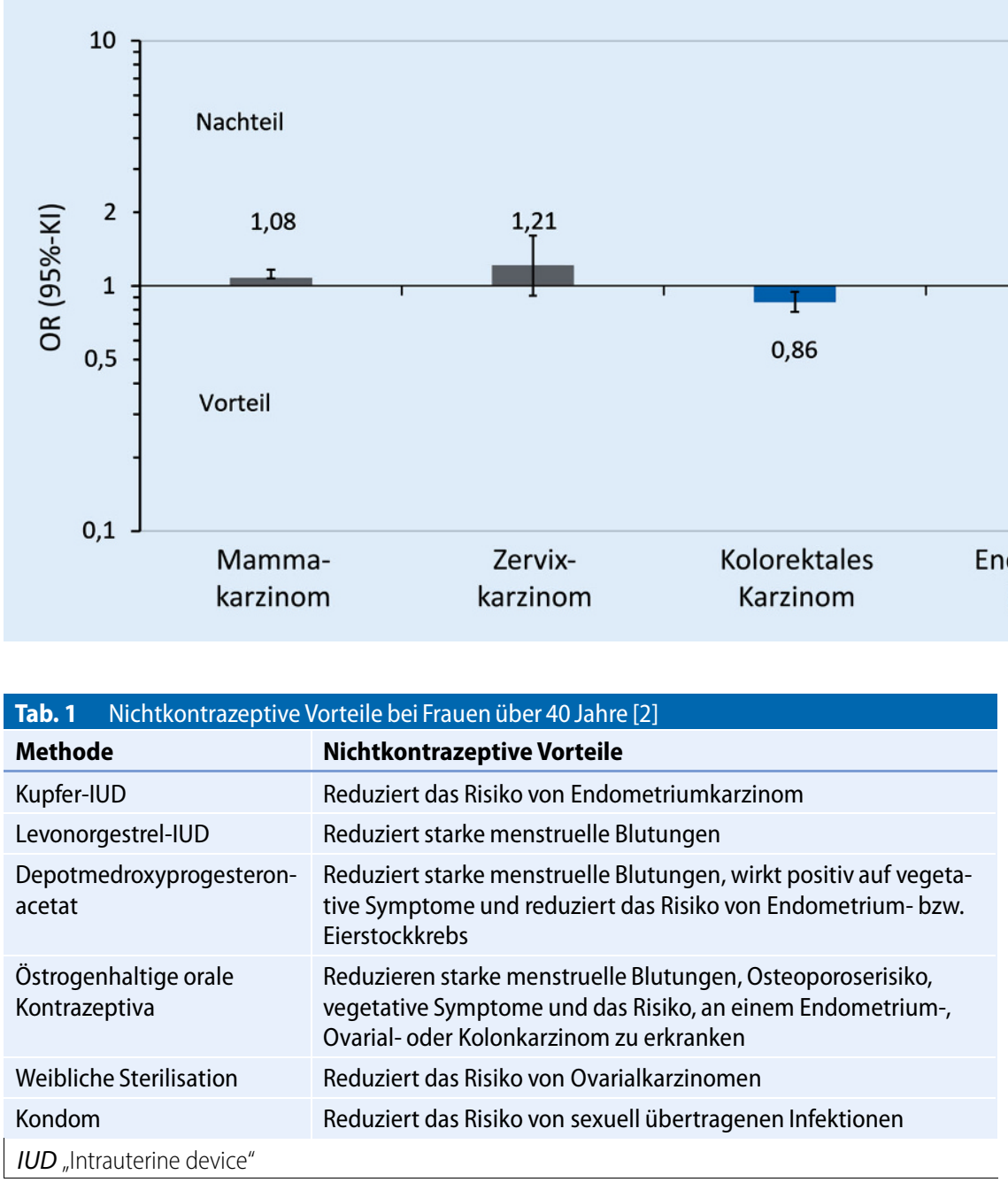




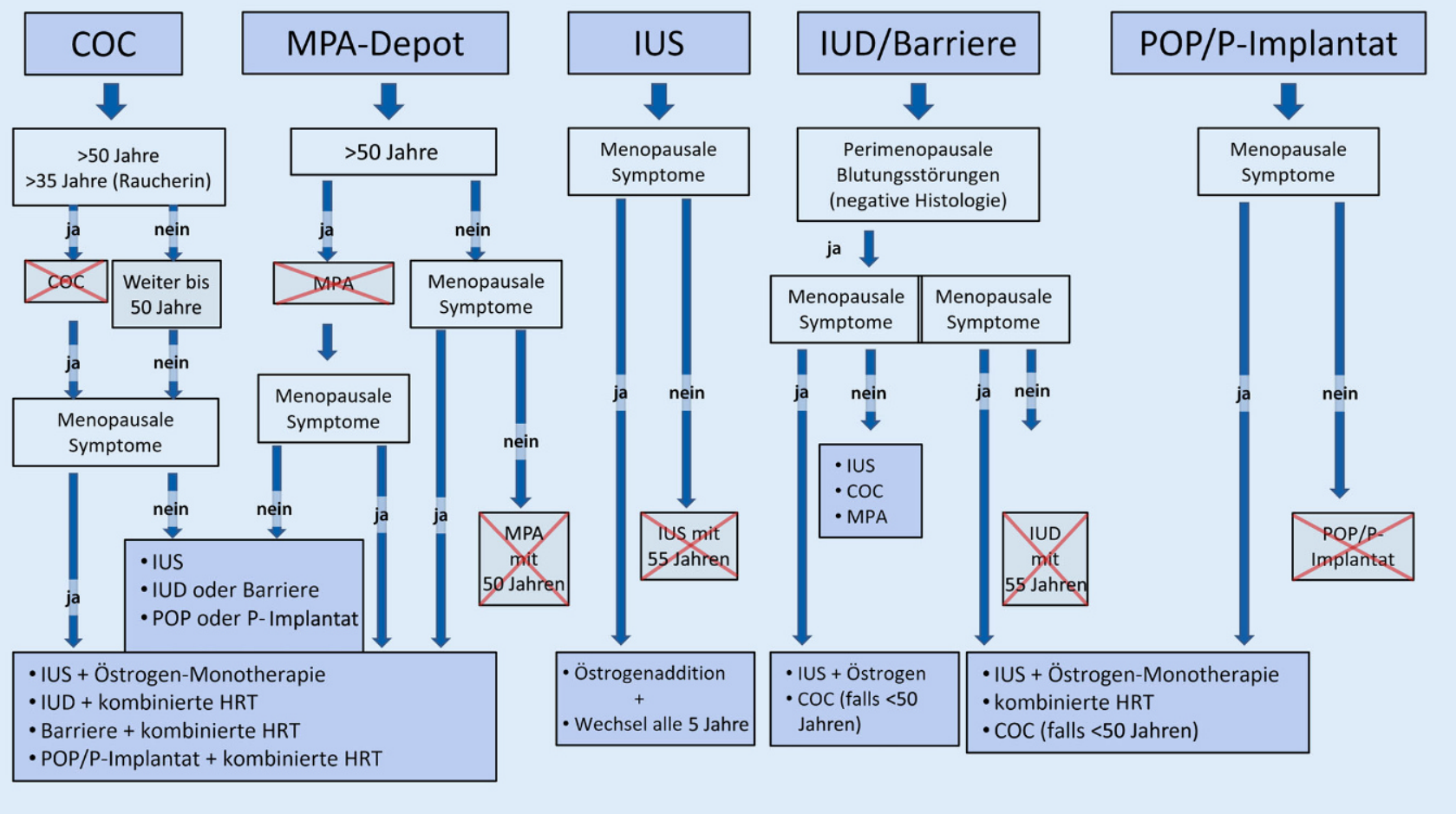

Abb. $5 \Delta$ Algorithmus zur Wahl des geeigneten Kontrazeptivums bei über 40-Jährigen [24]

lich der medizinischen Auswahlkriterien der Sicherheit der entsprechenden Kontrazeptiva. Die amerikanischen und britischen Gesellschaften haben jeweils ihre eigenen "guidelines“ angepasst [22-24]. Mittlerweile berufen sich fast alle ärztlichen Gesellschaften, die mit dem Thema befasst sind, auf die 2010 veröffentlichten und 2017 upgedateten Guidelines der britischen, NICE-akkreditierten Faculty of Sexual \& Reproductive Healthcare (FSRH). Entsprechend den Empfehlungen ist keine kontrazeptive Methode nur aufgrund des Alters kontraindiziert. Jedoch gibt es Erkrankungen, die bei älteren Frauen häufiger sind, und dadurch müssen dann bestimmte Methoden, insbesondere östrogenhaltige Kontrazeptiva, sehr vorsichtig angewandt werden. Klinisches Abwägen sollte immer eingesetzt werden, um die Vorteile und Nachteile der verschiedenen Kontrazeptiva in der Anwendung bei der jeweils ratsuchenden älteren Frau zu beleuchten. Um das Abwägen der geeigneten kontrazeptiven Methode für die ältere Frau zu erleichtern, wurde ein Algorithmus erarbeitet, abgeleitet von den Empfehlungen der FSRH (• Abb.5).

\section{Fazit für die Praxis}

\section{- Auch wenn die Fertilität im Alter} rückläufig ist, bleibt eine effektive Kontrazeption bei Frauen über 40 notwendig, um eine ungewollte Schwangerschaft zu vermeiden.

- Abgeleitet aus den vorhandenen internationalen Leitlinien, gibt es keine kontrazeptive Methode, die aufgrund des zunehmenden Alters abgelehnt werden muss.

- Effektive nichthormonelle oder ausschließlich progesteronhaltige Methoden sind für Frauen geeignet, die östrogenhaltige Kontrazeptiva meiden sollten.

- Frauen, die hormonelle Kontrazeptiva angewendet haben, können im Alter von 55 Jahren mit der Verhütung aufhören, da der Eintritt der Menopause angenommen werden kann.

\section{Korrespondenzadresse}

Prof. Dr. Helge Binder, FRANZCOG

Universitätsklinik für Gynäkologie, Geburtshilfe und Gynäkologische Endokrinologie, Kepler Universitätsklinikum GmbH, Med Campus IV. Krankenhausstraße 26-30, 4020 Linz,

Österreich

helge.binder@kepleruniklinikum.at

Funding. Open access funding provided by Kepler Universitätsklinikum Linz.

\section{Einhaltung ethischer Richtlinien}

Interessenkonflikt. H. Binder gibt an, dass kein Interessenkonflikt besteht.

Für diesen Beitrag wurden vom Autor keine Studien an Menschen oder Tieren durchgeführt. Für die aufgeführten Studien gelten die jeweils dort angegebenen ethischen Richtlinien.

Open Access. Dieser Artikel wird unter der Creative Commons Namensnennung 4.0 International Lizenz (http://creativecommons.org/licenses/by/4.0/deed. de) veröffentlicht, welche die Nutzung, Vervielfältigung, Bearbeitung, Verbreitung und Wiedergabe in jeglichem Medium und Format erlaubt, sofern Sie den/die ursprünglichen Autor(en) und die Quelle ordnungsgemäßnennen, einen Link zur Creative Commons Lizenz beifügen und angeben, ob Änderungen vorgenommen wurden. 


\section{Literatur}

1. Gold EB (2011) The timing of the Age at which natural menopause occurs. Obstet Gynecol Clin North Am 38(3):425-440

2. Allen RH et al (2013) Contraception in women over 40 years of age. CMAJ 185(7):565-573

3. Faculty of Sexual \& Reproductive Healthcare (FSRH) (2017) Guideline: Contraception for Women Aged Over 40 Years, firs published in July 2010, Updated November 2017. https://www.guidelinesinpractice.co. uk/womens-health/contraception-for-womenaged-over-40-what-when-and-for-how-long/ 453901.article

4. Harlow SD et al (2012) Executive summary of the Stages of Reproductive Aging Workshop + 10: addressing the unfinished agenda of staging reproductive aging, CLIMACTERIC 2012; Early Online, S1-10

5. Bundersministerium für Bildung und Frauen (2015) https://bildung.bmbwf.gv.at/frauen/ gender/gender index 2015.pdf

6. https://www.bpb.de/nachschlagen/zahlen-undfakten/soziale-situation-in-deutschland/61538/ altersgruppen

7. The Writing Group for the WHI Investigators (2002) Risks and benefits of estrogen plus progestin in healthy post-menopausal women: Principal results of the Women's Health Initiative randomized controlled trial. JAMA 288(3):321-333

8. The Women's Health Initiative Steering Committee (2004) Effects of Conjugated Equine Estrogen in Postmenopausal Women With Hysterectomy. The Women's Health Initiative Randomized Controlled Trial. JAMA 291:1701-1717

9. Stuenkel CA et al (2015) Treatment of symptoms of the menopause... Guideline. J Clin Endocrinol Metab 100(11):3975-4011

10. Beral V et al (2005) Endometrial cancer and hormone-replacement therapy in the Million Women Study. Lancet 365(9470):1543-1551

11. Spencer AL, Bonnema R, McNamara MC (2009) Helping women choose appropriate hormonal contraception: update on risks, benefits, and indications. Am J Med 122:497-506

12. Haider A, Shaw JC (2004) Treatment of acne vulgaris. JAMA 292:726-735

13. Lindh I, Ellstrom AA, Milsom I (2012) The effect of combined oral contraceptives and age on dysmenorrhoea: an epidemiological study. Hum Reprod 27:676-682

14. Gillum LA, Mamidipudi SK, Johnston SC (2000) Ischemic stroke risk with oral contraceptives: a meta-analysis. JAMA 284:72-78

15. Gillum LA, Mamidipudi SK, Review JSC (2001) current oral contraceptive use increases the risk for ischaemic stroke. Evid Based Med 6:60

16. Anonymous (1995) Venous thromboembolic disease and combined oral contraceptives: results of international multicentre case-control study. World Health Organization Collaborative Study of Cardiovascular Disease and Steroid Hormone Contraception. Lancet 346:1575-1582

17. Gierisch JM et al (2013) Oral contraceptive use and risk of ...cancers. Cancer Epidemiol Biomarkers Prev 22(11):1931-1943

18. Anonymous (1998) Cardiovascular disease and use of oral and injectable progestogenonly contraceptives and combined injectable contraceptives. Results of an international, multicenter, casecontrol study. World Health Organization Collaborative Study of Cardiovascular Disease and
Steroid Hormone Contraception. Contraception 57:315-324

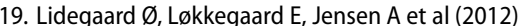
Thrombotic stroke and myocardial infarction with hormonal contraception. N Engl J Med 366:2257-2266

20. Kaunitz AM (2008) Clinical practice. Hormonal contraception in women of older reproductive age. NEngl J Med 358:1262-1270

21. ACOG (2006) ACOG practice bulletin no. 73 : use of hormonal contraception in women with coexisting medical conditions. Obstet Gynecol 107:1453-1472

22. ESHRE Capri Workshop Group (2009) Female contraception over 40. Hum Reprod Update 15(6):599-612

23. Anonymous (2010) US Centers for Disease Control and Prevention. US medical eligibility criteria for contraceptive use, 2010. MMWRMorb Mortal Wkly Rep 59(RR-4):1-86

24. Faculty of Sexual \& Reproductive Healthcare (FSRH) Contraception for Women Aged Over 40 Years, October 2017. https://www. fsrh.org/standards-and-guidance/documents/ fsrhguidance-contraception-for-women-agedover-40-years-2017/

Hinweis des Verlags. Der Verlag bleibt in Hinblick auf geografische Zuordnungen und Gebietsbezeichnungen in veröffentlichten Karten und Institutsadressen neutral.
Hier steht eine Anzeige. Springer 